\title{
Protostellar angular momentum evolution during gravoturbulent fragmentation
}

\author{
A.-K. Jappsen and R. S. Klessen
}

\author{
Astrophysikalisches Institut Potsdam, An der Sternwarte 16, 14482 Potsdam, Germany \\ e-mail: akjappsen@aip.de
}

Received 7 February 2004 / Accepted 26 April 2004

\begin{abstract}
Using hydrodynamic simulations we investigate the rotational properties and angular momentum evolution of prestellar and protostellar cores formed from gravoturbulent fragmentation of interstellar gas clouds. We find the specific angular momentum $j$ of molecular cloud cores in the prestellar phase to be on average $\langle j\rangle=7 \times 10^{20} \mathrm{~cm}^{2} \mathrm{~s}^{-1}$ in our models. This is comparable to the observed values. A fraction of those cores is gravitationally unstable and goes into collapse to build up protostars and protostellar systems, which then have $\langle j\rangle=8 \times 10^{19} \mathrm{~cm}^{2} \mathrm{~s}^{-1}$. This is one order of magnitude lower than their parental cores and in agreement with observations of main-sequence binaries. The loss of specific angular momentum during collapse is mostly due to gravitational torques exerted by the ambient turbulent flow as well as by mutual protostellar encounters in a dense cluster environment. Magnetic torques are not included in our models, these would lead to even larger angular momentum transport.

The ratio of rotational to gravitational energy $\beta$ in cloud cores that go into gravitational collapse turns out to be similar to the observed values. We find that $\beta$ is roughly conserved during the main collapse phase. This leads to the correlation $j \propto$ $M^{2 / 3}$, between specific angular momentum $j$ and core mass $M$. Although the temporal evolution of the angular momentum of individual protostars or protostellar systems is complex and highly time-variable, this correlation holds well in a statistical sense for a wide range of turbulent environmental parameters. In addition, high turbulent Mach numbers result in the formation of more numerous protostellar cores with, on average, lower mass. Therefore, models with larger Mach numbers result in cores with lower specific angular momentum. We find, however, no dependence on the spatial scale of the turbulence. Our models predict a close correlation between the angular momentum vectors of neighboring protostars during their initial accretion phase. Possible observational signatures are aligned disks and parallel outflows. The latter are indeed observed in some low-mass isolated Bok globules.
\end{abstract}

Key words. stars: formation - methods: numerical - hydrodynamics - turbulence - ISM: clouds

\section{Introduction}

Angular momentum plays a pivotal role in star formation. The amount of specific angular momentum determines whether a collapsing protostellar core will form a single star or a binary or higher-order multiple system.

Stars are thought to form by gravoturbulent fragmentation in interstellar clouds. The supersonic turbulence ubiquitously observed in molecular gas generates strong density fluctuations with gravity taking over in the densest and most massive regions. Once gas clumps become gravitationally unstable, collapse sets in and the central density increases until a protostellar object forms and grows in mass via accretion from the infalling envelope. Various aspects of the relation between supersonic turbulence and star formation have been discussed, e.g., by Hunter \& Fleck (1982), Elmegreen (1993), Larson (1995), Padoan (1995), Ballesteros-Paredes et al. (1999a,b, 2003), Padoan \& Nordlund (1999, 2002), Vázquez-Semadeni et al. (2000), Klessen et al. (2000), Heitsch et al. (2001), Klessen (2001a,b), Gammie et al. (2003), or
Vázquez-Semadeni et al. (2003). In particular see the reviews by Larson (2003) and Mac Low \& Klessen (2004).

This dynamic picture of gravoturbulent star formation challenges the so called "standard theory" where stars build up from the "inside-out" collapse of singular isothermal spheres, which are generally assumed to result from the quasistatic contraction of magnetically supported cloud cores due to ambipolar diffusion (Shu 1977; Shu et al. 1987). This picture, however, has always received strong criticism (e.g.,Whitworth \& Summers 1985; Whitworth et al. 1996; Nakano 1998). In particular, it seems strongly biased toward the formation of single stars (Whitworth et al. 1996) which is in contradiction to the observational fact that most (if not all) stars form as members of binary or higher-order multiple systems (see, e.g., the reviews by Bodenheimer et al. 2000; Mathieu et al. 2000, and references therein).

Gravitational collapse in the astrophysical context always involves solving the angular momentum problem. It results from the blatant discrepancy between the specific angular momentum observed in low-density gas on large scales and 
the amount of rotation present after collapse (Spitzer 1968; Bodenheimer 1995). The source of angular momentum on large scales lies in the differential rotation of the galactic disk and, closely related to that, on intermediate to small scales it results from the high degree of vorticity inextricably adherent to turbulent flows. The typical specific angular momentum $j$ of molecular cloud material, e.g., on scales of about $1 \mathrm{pc}$ is $j \approx 10^{23} \mathrm{~cm}^{2} \mathrm{~s}^{-1}$, while on scales of cloud cores, say below $0.1 \mathrm{pc}$, it is of order $10^{21} \mathrm{~cm}^{2} \mathrm{~s}^{-1}$. A binary $\mathrm{G}$ star with an orbital period of 3 days has $j \approx 10^{19} \mathrm{~cm}^{2} \mathrm{~s}^{-1}$, while the spin of a typical T Tauri star is a few $\times 10^{17} \mathrm{~cm}^{2} \mathrm{~s}^{-1}$. Our own Sun rotates only with $j \approx 10^{15} \mathrm{~cm}^{2} \mathrm{~s}^{-1}$. That means that during the process of star formation most of the initial angular momentum is removed from the collapsed object.

The presence of magnetic fields, in principle, provides a viable mechanism for locally reducing the angular momentum through magnetic braking. This was treated approximately by Ebert et al. (1960), and later calculated accurately by Mouschovias \& Paleologou (1979, 1980). The criterion for effective braking is essentially that the outgoing helical Alfvén waves from the rotating cloud have to couple to the ambient medium over a volume that contains roughly the same mass as the cloud itself. For the strong magnetic fields required by the standard theory of star formation, the deceleration time can be less than the free-fall time, leading to efficient transfer of angular momentum away from collapsing cores, and thus, to the formation of single stars. Field strengths small enough to allow for binary formation cannot provide support against collapse, thus pointing toward a more dynamic picture of star formation as offered by gravoturbulent fragmentation.

It is therefore a crucial test for any theory of star formation whether it can produce the required angular momentum loss during collapse while at the same time explain the high numbers of binaries and multiple stellar systems observed (e.g., Duquennoy \& Mayor 1991; Halbwachs et al. 2003). In a semiempirical analysis of isolated binary star formation Fisher (2004) presented the effects of turbulence in the initial state of the gas on binary orbital parameters. These properties were in agreement with observations if a significant loss of angular momentum was assumed. In the current investigation we focus on models of non-magnetic, supersonically turbulent, selfgravitating clouds and analyze the time evolution of angular momentum during formation and subsequent collapse of protostellar cores. Our main question is whether gravoturbulent fragmentation can solve or at least ease the so called "angular momentum" problem without invoking the presence of magnetic fields.

The structure of the paper is as follows. In Sect. 2 we introduce and discuss the numerical method of calculating the dynamical cloud evolution and the suite of models included in the current analysis. In Sect. 3 we present results on the angular momentum distribution of starless molecular cloud cores. We call them prestellar cores. Some of them collapse to become protostellar cores. In Sect. 4 we investigate the angular momentum evolution of their central protostellar objects. We report a statistical correlation between specific angular momentum and mass, and analyze its dependence on the turbulent environment. The angular momentum vectors of neighboring protostars tend to be aligned, at least in the early accretion phase. This is discussed in Sect. 5. Finally, in Sect. 6, we summarize and conclude.

\section{Models of dynamical cloud evolution}

\subsection{Numerical method}

To adequately describe the angular momentum evolution during gravoturbulent fragmentation, it is prerequisite to resolve formation and subsequent evolution of collapsing fragments over several orders of magnitude in density. Due to the stochastic nature of supersonic turbulence, it is not known in advance where and when local collapse will occur. We therefore resort to smoothed particle hydrodynamics (SPH) to solve the equations of hydrodynamics. It is a Lagrangian method with the fluid represented by an ensemble of particles and flow quantities obtained by local averaging (Benz 1990; Monaghan 1992). The method is able to resolve large density contrasts as particles are free to move and so naturally the particle concentration increases in high-density regions. Because it is computationally prohibitive to treat the cloud as a whole, we concentrate on subregions within the cloud and adopt periodic boundary conditions (Klessen 1997). Once the central region of a collapsing gas clump exceeds a density contrast of $\sim 10^{5}$, we introduce a "sink particle" (Bate et al. 1995), which accretes gas from its surrounding and keeps track of mass and linear and angular momentum. Replacing the high-density cores with "sink particles" allows us to follow the angular momentum evolution of collapsed cores over many free-fall times. Altogether, the performance and convergence properties of the method are well understood and tested against analytic models and other numerical schemes in the context of turbulent supersonic astrophysical flows. For a detailed discussion the reader is referred to Bate \& Burkert (1997), Mac Low et al. (1998), Lombardi et al. (1999), Klessen \& Burkert (2000, 2001) and Klessen et al. (2000).

\subsection{Models of turbulent self-gravitating clouds}

The large observed linewidths in molecular clouds imply the presence of supersonic velocity fields that carry enough energy to counterbalance gravity on global scales (see, e.g., the review by Williams et al. 2000). However, it is known that turbulent energy dissipates rapidly, roughly on the free-fall timescale (Mac Low et al. 1998; Stone et al. 1998; Padoan \& Nordlund 1999). Unlike previously thought, this is independent of the presence of magnetic fields. Magnetic fields also do not significantly alter the efficiency of local collapse for driven turbulence (Heitsch et al. 2001). To a first approximation, the fields are therefore taken as being dynamically unimportant in our current models, and are not included. We also disregard possible feedback effects from the star formation process itself (like bipolar outflows, stellar winds, or ionizing radiation from newborn O or B stars). Our analysis of angular momentum evolution of protostellar cores therefore focuses on the process of gravoturbulent fragmentation, i.e. on the interplay between turbulence and self-gravity only. 
Table 1. Sample parameters, name of the environment used in the text consisting of the Mach number $\mathcal{M}$ and the driving scale $k$ (GA denotes the model with Gaussian density), number $\mathcal{N}$ of protostellar objects (i.e. "sink particles" in the centers of protostellar cores) at the final stage of the simulation, percentage of accreted mass at the final stage $M_{\text {acc }} / M_{\text {tot }}$, parameter $\mathcal{A}$ see Eq. (5), parameter $\mathcal{B}$ see Eq. (9).

\begin{tabular}{lllllll}
\hline \hline Name & $k$ & $\mathcal{M}$ & $\mathcal{N}$ & $\begin{array}{l}M_{\text {acc }} / M_{\text {tot }} \\
{[\%]}\end{array}$ & $\begin{array}{l}\mathcal{A} \\
{\left[10^{20}\right.}\end{array}$ & $\begin{array}{l}\mathcal{B} \\
\mathrm{cm}^{2} \mathrm{~s}^{-1}\end{array}$ \\
\hline M2.0k2 & $1 . .2$ & 2.0 & 68 & 75 & 1.7 & 1.7 \\
M2.0k4 & $3 . .4$ & 2.0 & 62 & 48 & 2.0 & 2.0 \\
M2.0k8 & $7 . .8$ & 2.0 & 11 & 66 & 1.7 & 1.6 \\
M3.2k2 & $1 . .2$ & 3.2 & 62 & 80 & 1.2 & 1.3 \\
M3.2k4 & $3 . .4$ & 3.2 & 37 & 82 & 2.0 & 1.9 \\
M3.2k8 & $7 . .8$ & 3.2 & 17 & 60 & 2.7 & 2.6 \\
M6k2 & $1 . .2$ & 6.0 & 110 & 76 & 1.3 & 1.3 \\
M6k4 & $3 . .4$ & 6.0 & 60 & 65 & 1.5 & 1.7 \\
M6k8 & $7 . .8$ & 6.0 & 7 & 4 & 1.9 & 1.4 \\
M10k2 & $1 . .2$ & 10.0 & 100 & 38 & 1.0 & 1.0 \\
M10k4 & $3 . .4$ & 10.0 & 10 & 6 & 2.0 & 1.4 \\
M10k8 & $7 . .8$ & 10.0 & 27 & 8 & 1.4 & 1.05 \\
GA & $\ldots$ & $\ldots$ & 56 & 85 & 1.4 & 1.05 \\
\hline
\end{tabular}

The suite of models considered here consists of 12 numerical simulations where turbulence is maintained with constant root-mean-square Mach numbers in the range $2 \leq \mathcal{M} \leq 10$. This roughly covers the range observed in typical Galactic molecular clouds. We apply a non-local scheme that inserts energy in a limited range of wavenumbers at a given rate (Mac Low 1999). We distinguish between turbulence that carries its energy mostly on large scales, at wavenumbers $1 \leq k \leq$ 2 , on intermediate scales, i.e. $3 \leq k \leq 4$, and on small scales with $7 \leq k \leq 8$. The corresponding wavelengths are $\ell=L / k$, where $L$ is the total size of the computed volume. The models are labeled mnemonically as MMkk, with rms Mach number $\mathcal{M}$ and wavenumber $k$. Each of these simulations contains 205379 SPH particles. We also consider a model that is globally unstable and contracts from Gaussian initial conditions without turbulence (for details see Klessen \& Burkert 2000, 2001). It is called GA and was run with 500000 particles. The main parameters are summarized in Table 1 . Note that the final star formation efficiency varies between the different models, as indicated in Col. 5 of Table 1. This simply reflects the evolutionary stage at the time when we stop the calculation. In some cases the accretion timescale is too long to follow the simulation to high efficiencies.

\subsection{Physical scaling and naming convention}

The models are computed in normalized units using an isothermal equation of state. Scaled to physical units we adopt a temperature of $11.3 \mathrm{~K}$ corresponding to a sound speed $c_{\mathrm{s}}=$ $0.2 \mathrm{~km} \mathrm{~s}^{-1}$, and we use a mean density of $n\left(\mathrm{H}_{2}\right)=10^{5} \mathrm{~cm}^{-3}$, which is typical for star-forming molecular cloud regions (e.g. in $\rho$-Ophiuchi, see Motte et al. 1998). The mean thermal Jeans mass $^{1}$ in all models is $\left\langle M_{\mathrm{J}}\right\rangle=1 M_{\odot}$. The turbulent models contain a mass of $120 M_{\odot}$ within a cube of size $0.28 \mathrm{pc}$, and the Gaussian model has $220 M_{\odot}$ in a volume of $(0.34 \mathrm{pc})^{3}$. The global free-fall timescale is $\tau_{\mathrm{ff}}=10^{5} \mathrm{yr}$, and the simulations cover a density range from $n\left(\mathrm{H}_{2}\right) \approx 100 \mathrm{~cm}^{-3}$ in the lowest density regions to $n\left(\mathrm{H}_{2}\right) \approx 10^{9} \mathrm{~cm}^{-3}$ where the central parts of collapsing gas clumps are converted into "sink particles".

Each "sink particle" defines a control volume with a fixed radius of $560 \mathrm{AU}$. We cannot resolve the subsequent evolution in its interior. After $\sim 10^{3} \mathrm{yr}$ a protostar will form in the very center. Because of angular momentum conservation most of the matter that falls in will assemble in a protostellar disk. It is then transported inward by viscous and possibly gravitational torques (e.g., Bodenheimer 1995; Papaloizou \& Lin 1995; Lin \& Papaloizou 1996). With typical disk sizes of the order of several hundred AU, the control volume fully encloses both star and disk. If low angular momentum material is accreted, the disk is stable and most of the material ends up in the central star. In this case, the disk simply acts as a buffer and smooths eventual accretion spikes. It will not delay or prevent the mass growth of the central star by much. However, if material that falls into the control volume carries large specific angular momentum, then the mass load onto the disk is likely to be faster than the inward transport. The disk grows large and may become gravitationally unstable and fragment. This will lead to the formation of a binary or higher-order multiple (Bodenheimer et al. 2000).

Throughout this paper, we adopt the following naming convention: in the pre-collapse phase, we call high-density gas clumps prestellar cores or simply gas clumps. They build up at the stagnation points of convergent flows. The flows result from turbulent motion that establishes a complex network of interacting shocks. The gas clumps are identified and characterized using a three-dimensional clump-finding algorithm as described in Appendix A of Klessen \& Burkert (2000). The fluctuations in turbulent velocity fields are highly transient. They can disperse again once the converging flow fades away. Even clumps that are strongly dominated by gravity may get disrupted by the passage of a new shock front. For local collapse to result in the formation of stars, Jeans-unstable, shockgenerated density fluctuations therefore must collapse to sufficiently high densities on time scales shorter than the typical time interval between two successive shock passages. We include in our analysis only Jeans-unstable gas clumps. Angular momentum is calculated from the internal motions with respect to the location of the density maximum. These objects correspond to the so called starless cores observed e.g. by Goodman et al. (1993), Barranco \& Goodman (1998), Jijina et al. (1999), and others. They are thought to collapse and build up a central protostar or protostellar system in the later stages of evolution. Once collapse has led to the formation of an embedded protostar (in our scheme identified by a central "sink particle") we call the object protostellar core or also protostar. The angular

\footnotetext{
${ }^{1}$ We use a spherical definition of the Jeans mass, $M_{\mathrm{J}} \equiv 4 / 3 \pi \rho \lambda_{\mathrm{J}}^{3}$, with density $\rho$ and Jeans length $\lambda_{\mathrm{J}} \equiv\left(\frac{\pi \mathcal{R} T}{G \rho}\right)^{1 / 2}$ and where $G$ and $\mathcal{R}$ are the gravitational and the gas constant. The mean Jeans mass $\left\langle M_{\mathrm{J}}\right\rangle$ is then determined from the average density in the system $\langle\rho\rangle$.
} 


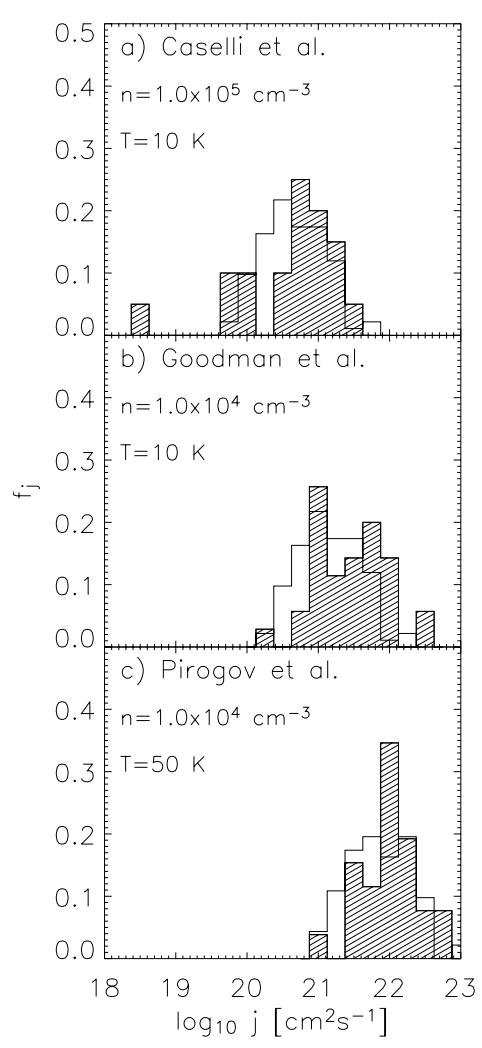

Fig. 1. The distribution of specific angular momenta of prestellar cores formed in our simulations using model M6k2 (non-hatched histogram) is compared to the distribution of specific angular momenta of observed molecular cloud cores (hatched distributions). The observational data were taken in a) from Table 5 in Caselli et al. (2002), in b) from Table 2 in Goodman et al. (1993), Table 4 in Barranco \& Goodman (1998) and Table A2 in Jijina et al. (1999) and in c) from Table 7 in Pirogov et al. (2003). We take $f_{j}$ to represent the percentage of the total number of existing cores in a specific angular momentum bin.

momentum is obtained as the spin accumulated by the "sink particle" during its accretion history. The distribution is best compared with observations of main-sequence binaries as we expect the unresolved star-disk system interior to the "sink particle" to break up into a binary or higher-order multiple.

\section{Molecular cloud clumps and prestellar cores}

Figure 1 shows the distribution of the specific angular momenta of the gas clumps that were identified in the turbulent environment M6k2 (see Table 1). We compare with observational values taken from Caselli et al. (2002) for a), from Goodman et al. (1993), Barranco \& Goodman (1998), Jijina et al. (1999) for b) and from Pirogov et al. (2003) for c). According to Goodman et al. (1993) the values for the specific angular momenta are obtained by using best-fit velocity gradients from maps of observed line-center velocities under the assumption of solid body rotation. The cores in Goodman et al. (1993), Barranco \& Goodman (1998), Jijina et al. (1999) were mapped in the $(J, K)=(1,1)$ transition of $\mathrm{NH}_{3}$, whereas the massive cloud cores in Pirogov et al. (2003) and the dense cloud cores in Caselli et al. (2002) were mapped in $\mathrm{N}_{2} \mathrm{H}^{+}$. As found in
Caselli et al. (2002) the two molecular species trace essentially the same material, especially in starless cores. Model clumps and observed molecular cloud clumps have comparable mass spectra (Klessen 2001a) and similar shapes (Klessen \& Burkert 2000; Ballesteros-Paredes et al. 2003). Figure 1 demonstrates that something similar holds for the distribution of specific angular momenta.

Note that, when transforming from dimensionless code units into physical scales, the specific angular momentum depends on the mean density $n$ and the temperature $T$ as $j \propto$ $T / \sqrt{n\left(\mathrm{H}_{2}\right)}$. In Fig. 1c, we use our standard scaling corresponding to regions like the $\rho$ Ophiuchi main cloud (Motte et al. 1998). This is adequate for the low-mass cores studied by Caselli et al. (2002), and in Sect. 4 we will furthermore show that the specific angular momenta of collapsed cores then fall into the right range for main-sequence binaries.

We find that the specific angular momenta of prestellar cores have values between $1 \times 10^{20} \mathrm{~cm}^{2} \mathrm{~s}^{-1}$ and $5 \times 10^{21} \mathrm{~cm}^{2} \mathrm{~s}^{-1}$ with a mean value of approximately $\langle j\rangle \approx 5 \times 10^{20} \mathrm{~cm}^{2} \mathrm{~s}^{-1}$. This is in good agreement with the Caselli et al. (2002) sample which has $\langle j\rangle=7 \times 10^{20} \mathrm{~cm}^{2} \mathrm{~s}^{-1}$. Their cloud cores have a mean mass of $\sim 6 M_{\odot}$, comparable to the core masses in our simulations. A Kolmogorov-Smirnov (KS) test was performed. We find that at the $50 \%$ level the distributions are statistically indistinguishable.

However, the cores observed by Goodman et al. (1993) and Pirogov et al. (2003) trace lower densities and have higher mean masses of around $50 M_{\odot}$ and $500 M_{\odot}$, respectively. In Fig. 1b we therefore use $n\left(\mathrm{H}_{2}\right)=1 \times 10^{4} \mathrm{~cm}^{-3}$ and $T=10 \mathrm{~K}$ leading to $\langle j\rangle=1.5 \times 10^{21} \mathrm{~cm}^{2} \mathrm{~s}^{-1}$. This matches the observations, since Goodman et al. (1993) find $\langle j\rangle=6 \times 10^{21} \mathrm{~cm}^{2} \mathrm{~s}^{-1}$ and a median value of $3 \times 10^{21} \mathrm{~cm}^{2} \mathrm{~s}^{-1}$. The massive cores mapped by Pirogov et al. (2003) have higher velocity dispersions, higher kinetic temperatures $(20-50 \mathrm{~K})$ and densities $n\left(\mathrm{H}_{2}\right) \geq 1 \times 10^{4} \mathrm{~cm}^{-3}$. The resulting mean specific angular momentum is $\langle j\rangle=1.5 \times 10^{22} \mathrm{~cm}^{2} \mathrm{~s}^{-1}$. Again, adequate scaling in Fig. 1a results in higher values of $j$ in our models and leads to better agreement with the observed distribution.

Given the simplified assumptions in our numerical models, we find remarkably good agreement with the observed specific angular momenta in the prestellar phase. Similar findings are reported by Gammie et al. (2003). Similar to our study, they follow the dynamical evolution of an isothermal, self-gravitating, compressible, turbulent ideal gas. However, they include the effects of magnetic fields and solve the equations of motion using a grid-based method (the well-known ZEUS code). Their approach is thus complementary to ours. The $j$ distribution that results from their simulations peaks at $4 \times 10^{22} \mathrm{~cm}^{2} \mathrm{~s}^{-1}$. They fix the mean number density at $n\left(\mathrm{H}_{2}\right) \approx 1.0 \times 10^{2} \mathrm{~cm}^{-3}$ and use $T=10 \mathrm{~K}$. Using the same physical scaling we get very similar values, i.e. $\langle j\rangle=4 \times 10^{22} \mathrm{~cm}^{2} \mathrm{~s}^{-1}$.

This mean value also falls in the range of specific angular momenta of cores that form in simulations by $\mathrm{Li}$ et al. (2004). They also use a version of the ZEUS code (ZEUS-MP) to perform high-resolution, three-dimensional, super-Alfvénic turbulent simulations to investigate the role of magnetic fields in self-gravitating core formation within turbulent molecular clouds. Adopting the same physical scaling as in 


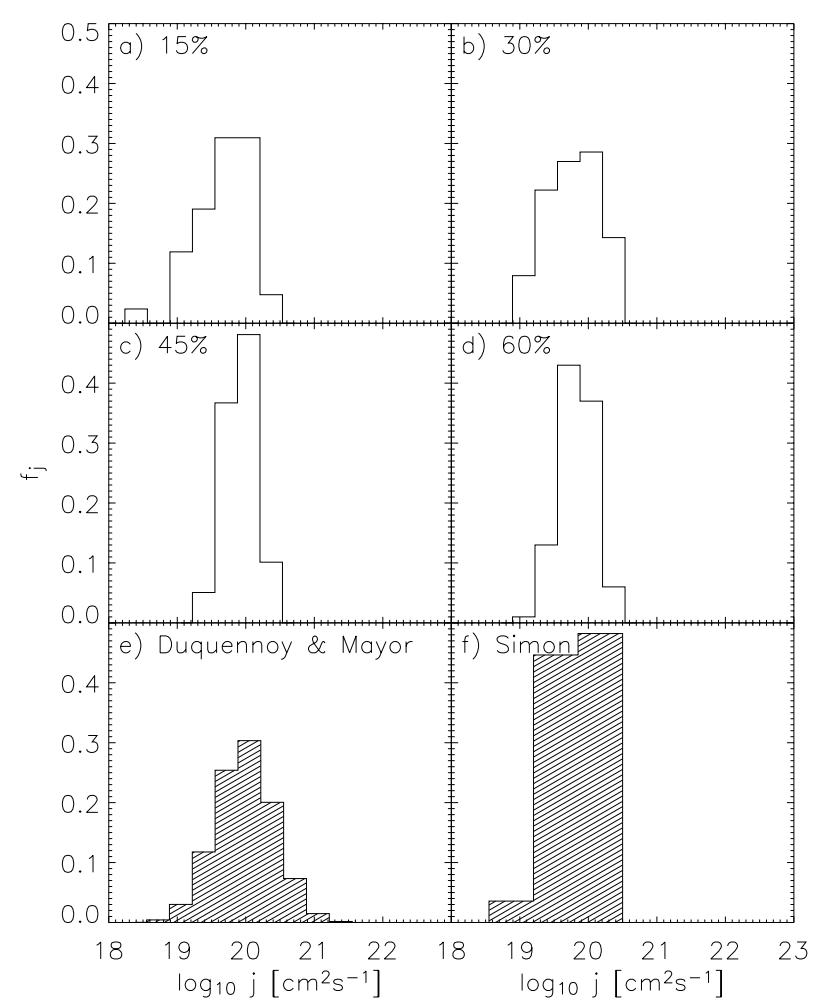

Fig. 2. Distribution of specific angular momenta of the protostars or protostellar systems at different evolutionary phases of the numerical model M6k2 as denoted by the local star formation efficiency in percent, a)-d). We compare in e) with the $j$-distribution of binaries among nearby G-dwarf stars from Duquennoy \& Mayor (1991) (for details see text) and in f) with the distribution of specific angular momenta of binaries in the Taurus star-forming region from Fig. 5 in Simon (1992). Again, $f_{j}$ represents the normalized distribution function.

Gammie et al. (2003), the specific angular momentum of their cores takes values between $5 \times 10^{21} \mathrm{~cm}^{2} \mathrm{~s}^{-1}$ and $8 \times 10^{22} \mathrm{~cm}^{2} \mathrm{~s}^{-1}$.

\section{Protostars and protostellar systems}

Figure 2 shows the distribution of specific angular momenta of collapsed cores at four different stages of mass accretion, ranging from $15 \%$ of the total available mass in the molecular cloud accreted onto collapsed cores in Fig. 2a to 60\% in Fig. 2d. While the distribution narrows during the evolution, the mean specific angular momentum remains essentially at the same value $j=(8 \pm 2) \times 10^{19} \mathrm{~cm}^{2} \mathrm{~s}^{-1}$ with a range from $10^{18} \mathrm{~cm}^{2} \mathrm{~s}^{-1}$ to $5 \times 10^{20} \mathrm{~cm}^{2} \mathrm{~s}^{-1}$. The specific angular momenta of the protostellar cores in the considered model are approximately one order of magnitude smaller than the ones of the Jeans-unstable clumps, but both distributions join without a gap. In a statistical sense, there is a continuous transition as loss of angular momentum occurs during contraction. The range of specific angular momenta of the protostellar cores agrees well with the observed values for binaries (e.g., Bodenheimer 1995). For this reason we compare in Figs. 2a-d the model distributions with observations of binaries among G-dwarf stars by Duquennoy \& Mayor (1991) in Fig. 2e and with observations of young binaries in the Taurus star forming region by Simon (1992) in Fig. 2f.

Duquennoy \& Mayor (1991) derived a Gaussian-type period distribution for their sample. Based on this distribution we calculated the distribution of the specific angular momenta using the following equation (see also Kroupa 1995b, Eq. (10)):

$j=6.23 \times 10^{18}\left(1-e^{2}\right)^{1 / 2} P^{1 / 3} \frac{m_{1} m_{2}}{\left(m_{1}+m_{2}\right)^{4 / 3}}\left(\mathrm{~cm}^{2} \mathrm{~s}^{-1}\right)$

where masses are in $M_{\odot}$ and period $P$ is in days. We used a primary mass $m_{1}$ of $1 M_{\odot}$, a mean mass ratio between primary and secondary $q=m_{2} / m_{1}=0.25$ and a mean eccentricity $e=0.31$.

The resulting distribution has a mean specific angular momentum of $1.6 \times 10^{20} \mathrm{~cm}^{2} \mathrm{~s}^{-1}$, which agrees well with the values from the simulations. This also holds for Fig. $2 \mathrm{f}$ which was taken from Fig. 5 in Simon (1992). The mean specific angular momentum here has a value of $1.6 \times 10^{20} \mathrm{~cm}^{2} \mathrm{~s}^{-1}$. The agreement of the distributions was confirmed by a $\chi^{2}$ statistical test. Since our numerical resolution is not sufficient to follow the subfragmentation of collapsing cores into binary or higherorder multiple systems, the time evolution of $j$ is an important tool to evaluate our models. We see a clear progression from the rotational properties of gas clumps (as discussed in Sect. 3) to those of the resulting collapsed cores. A similar correlation is observed between cloud cores (Caselli et al. 2002) and typical main-sequence binaries (Duquennoy \& Mayor 1991). The former may be the direct progenitors of the latter.

\subsection{Example of the angular momentum evolution of a protostellar core in a cluster}

The evolution of the specific angular momenta of individual protostellar cores can be very complex depending on the rotational properties of their environment. There is a strong connection to the time evolution of the mass accretion rate.

In Fig. 3 we select five collapsed cores in model M6k2 with about the same final mass. All of them show a similar evolution of the specific angular momentum with increasing mass (Fig. 3a) and time (Fig. 3b). Nevertheless there are visible differences in the details.

Initially, the specific angular momentum increases with growing mass. However, at later stages the evolution strongly depends on the secular properties of the surrounding flow. In cores 43,96 , and 101, for example, $j$ decreases again after reaching a peak value, while for cores 17 and $47 j$ stays close to the maximum value. Depending on the specific angular momentum of the accreted material, the resulting protostellar disks are expected to evolve quite differently. For example, preliminary 2-dimensional hydrodynamic calculations show that core 17 and core 47 will probably develop a stable disk (Bodenheimer 2003, private communication). The ratio of rotational to gravitational energy for the peak value of $j$ is $\beta=0.005$ for core 17 and $\beta=0.003$ for core 43 . On the other hand, core 101 will fragment into a binary star. It has a $\beta=0.016$. Also, the disk of core 96 is highly unstable with corresponding $\beta=0.016$. The evolution of core 43 has not yet been followed sufficiently long to determine whether it will 

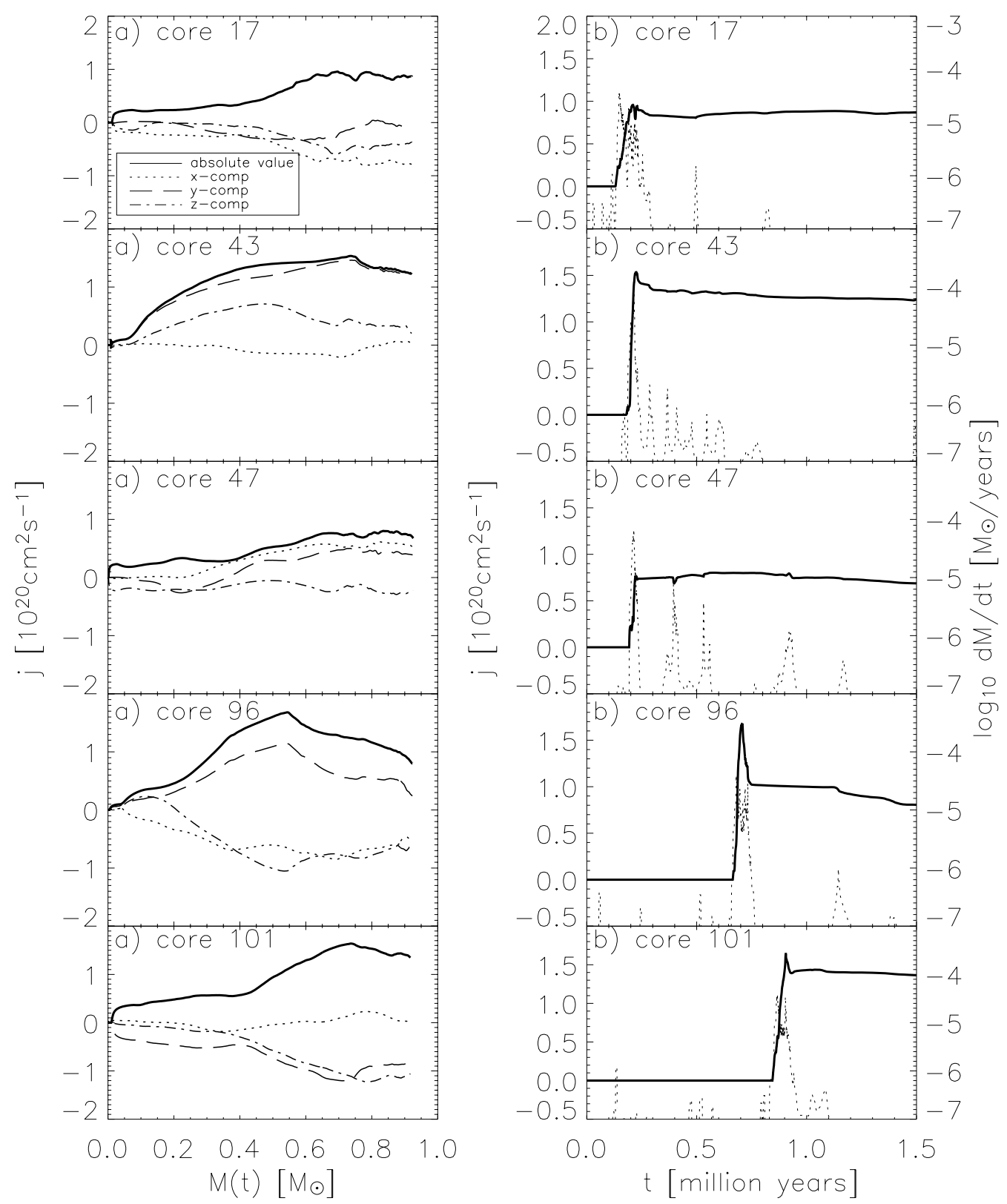

Fig. 3. Absolute value of specific angular momentum (solid line) of our model M6k2 as a) a function of mass and as b) a function of time for five different protostellar objects with approximately equal final masses $\left(M=0.94 M_{\odot}\right)$. In a) the $x$-component (dotted line), the $y$-component (dashed line) and the z-component (dashed-dotted line) of the specific angular momenta are shown as well. For comparison the mass accretion rates onto the protostar are indicated in $\mathbf{b}$ ) by a dashed line (associated y-axis on the right hand side).

fragment to form a binary star or not. These results show the importance of the specific angular momentum on the evolution of the protostellar object. A high value $\beta>0.01$ leads to fragmentation whereas $\beta<0.01$ results in a stable disk. A similar result was found by Boss (1999) for slowly rotating, magnetic clouds.

Figure $3 \mathrm{~b}$ shows that the change in specific angular momentum is closely linked to the mass accretion. At the point in time where the mass accretion rate (dotted line) has a pronounced peak, the change in specific angular momentum is also significant. A high mass accretion rate can result in an increase of specific angular momentum. But as seen for core 96 in Fig. $3 \mathrm{~b}$ a high mass accretion rate can also lead to a reduction of specific angular momentum. The exact evolution of the specific angular momentum is thus closely linked to the flow properties of the surrounding material.

\subsection{Statistical correlation between specific angular momentum and mass}

As mentioned above we find a correlation between mass $M$ and specific angular momentum $j$ in a statistical sense. The result is depicted in Fig. 4. It shows the angular momentum evolution as a function of mass for all 110 collapsed cores in model M6k2.

In Fig. 4a, following Goodman et al. (1993) we adopt rigid body rotation with constant angular velocity $\Omega$ and uniform 


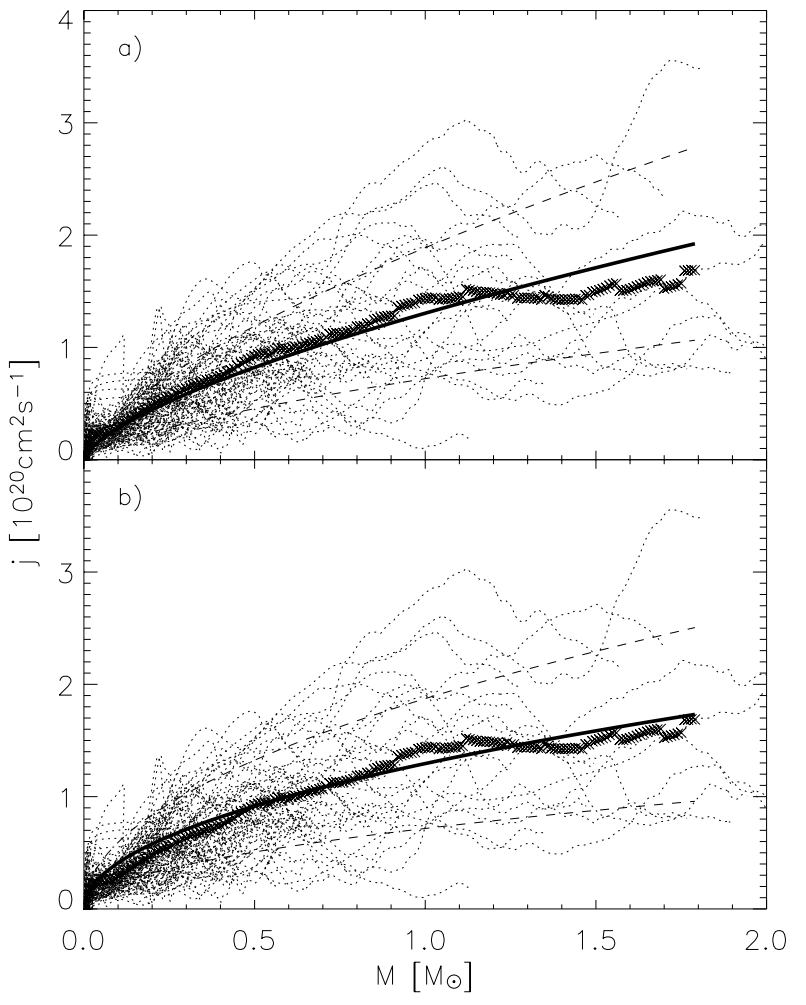

Fig. 4. Absolute values of specific angular momenta (dotted lines) of all protostars (i.e. "sink particles") from our model M6k2 as a function of mass. The specific angular momenta are averaged at certain mass values which are separated by $0.01 M_{\odot}$ and the resulting points are indicated by crosses. The solid line represents a fit of these averaged specific angular momenta in the mass range between 0 and $1.7 M_{\odot}$ In a) we fit with a function of the form: $j=\mathcal{A}\left(M / M_{\odot}\right)^{2 / 3}$, $\mathcal{A}=(1.3 \pm 0.6) \times 10^{20} \mathrm{~cm}^{2} \mathrm{~s}^{-1}$, and in $\left.\mathbf{b}\right)$ we use a square root function: $j=\mathcal{B} \sqrt{M / M_{\odot}}, \mathcal{B}=(1.3 \pm 0.6) \times 10^{20} \mathrm{~cm}^{2} \mathrm{~s}^{-1}$. One standard deviation is marked by the dashed lines.

core density $\rho$. With these assumptions the specific angular momentum $j$ can be written as:

$j=p \Omega R^{2}$.

For a uniform density sphere $p=\frac{2}{5}$. The mass $M$ of a sphere with constant density $\rho_{0}$ is related to the radius $R$ via:

$M=\frac{4 \pi}{3} \rho_{0} R^{3}$.

From Eqs. (2) and (3) follows that $j$ can be expressed as:

$j=p \Omega\left(\frac{3}{4 \pi \rho_{0}}\right)^{2 / 3} M^{2 / 3}$.

Therefore we fit the average angular momentum with a function of the form:

$j=\mathcal{A}\left(M / M_{\odot}\right)^{2 / 3}$,

where $\mathcal{A}=p \Omega\left(\frac{3 M_{\odot}}{4 \pi \rho_{0}}\right)^{2 / 3}$. From Fig. 4 a the constant $\mathcal{A}$ has a value of $(1.3 \pm 0.6) \times 10^{20} \mathrm{~cm}^{2} \mathrm{~s}^{-1}$. This fit formula can be applied to different turbulent cloud environments, and we list the corresponding values of $\mathcal{A}$ for our model suite in Table 1 . Using the fitted value and the density $\rho_{0}=4 \times 10^{-15} \mathrm{~g} \mathrm{~cm}^{-3}$ where protostellar cores are identified, we calculate an angular velocity $\Omega=1.33 \times 10^{-11} \mathrm{~s}^{-1}$.

In this picture the ratio of rotational to gravitational energy $\beta$ can be written as:

$\beta=\frac{(1 / 2) I \Omega^{2}}{q G M^{2} / R}=\frac{3 p}{8 \pi q} \frac{\Omega^{2}}{\rho_{0} G}$,

where the moment of inertia is given by $I=p M R^{2}$, and $q=\frac{3}{5}$ is defined such that $q G M^{2} / R$ represents the gravitational potential of a uniform density sphere. With the assumptions of constant angular velocity and uniform density it follows that $\beta$ is also constant. With $\Omega$ and $\rho_{0}$ as above, we get values $\beta \approx 0.05$.

Goodman et al. (1993) as well as Burkert \& Bodenheimer (2000) derived scaling relations where $\beta$ is independent of radius. Similar values for $\beta$ were also found by Goodman et al. (1993) for the observed cloud cores. In good agreement with our calculations they found that all values are below 0.18 with the majority under 0.05 .

The fit in Fig. 4a rests on the assumption of the collapse of an initially homogeneous sphere with constant angular velocity. Using "sink particles" however, which have a constant radius, makes it necessary to examine another possibility. In Fig. 4b we thus assume a constant radius $R$ and a constant $\beta$. Choosing a constant $\beta$ is supported by the observations as discussed above and by our simulations as we show below. With $\beta$ and Eq. (6) (which still holds) it follows that the angular velocity depends on the density as

$\Omega=\sqrt{\frac{8 \pi q}{3 p} G \rho \beta}$.

Thus, the angular velocity $\Omega$ is no longer a constant. This implies for the specific angular momentum $j$ :

$j=p \Omega R^{2}=\sqrt{2 p R q G \beta} \sqrt{M}$.

Following Eq. (8) we fit our data in Fig. $4 \mathrm{~b}$ with a square root function:

$j=\mathcal{B} \sqrt{M / M_{\odot}}$,

where, again, the moment of inertia is given by $I=p M R^{2}$, and $q=\frac{3}{5}$ is defined such that $q G M^{2} / R$ represents the gravitational potential of a uniform density sphere. With the above density and angular velocity Eq. (6) results in $\beta \approx 0.05$. For the new fit we find a scaling factor $\mathcal{B}=(1.3 \pm 0.6) \times 10^{20} \mathrm{~cm}^{2} \mathrm{~s}^{-1}$ in the mass range $0 \leq M / M_{\odot} \leq 1.7$. Again, this fit formula can be applied to different turbulent cloud environments, and we list the corresponding values of $\mathcal{B}$ for our model suite in Table 1.

The question remains if our simulations support the assumption of a constant $\beta$. In Fig. 5 we compare the distribution of $\beta$ measured by Goodman et al. (1993) with values we extract from our model. For the prestellar cores we use the definition

$\beta=\frac{E_{\mathrm{rot}}}{E_{\text {grav }}}$,

and calculate rotational and potential energy, $E_{\mathrm{rot}}$ and $E_{\mathrm{grav}}$, consistently from the full three-dimensional gas distribution of 


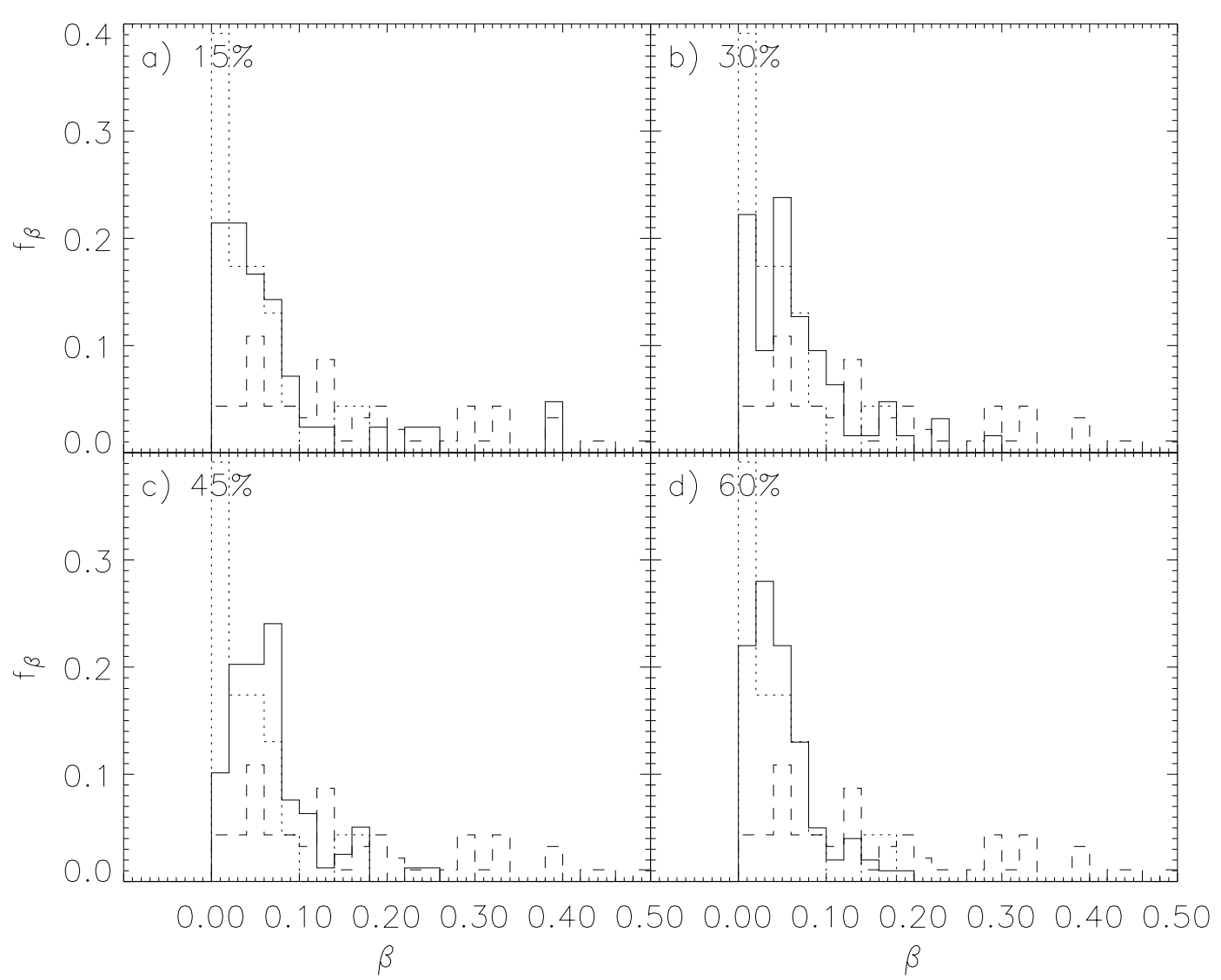

Fig. 5. Distribution of $\beta$ obtained from our model M6k2 with $15 \%$ a), $30 \%$ b), $45 \%$ c) and $60 \%$ d) of the material accreted. For the collapsed protostellar cores in our models (solid lines) we calculate $\beta$ using Eq. (6), where we assume solid-body rotation of a uniform density sphere and take the calculated specific angular momentum $j$. For the prestellar cores (i.e. Jeans-unstable gas clumps; see the dashed lines) we derive $\beta$ self-consistently from the three-dimensional density and velocity structure, using Eq. (10). For comparison we also indicate with dotted lines the values reported by Goodman et al. (1993, see their Fig. 11) for observed molecular cloud cores. These were obtained with the same assumptions, and we use the same binning with $f_{\beta}$ representing the fraction of objects per $\beta$ bin.

each gas clump. We do not adopt any assumption about symmetry and shape of the density and velocity structure. If we assume that each clump is spherical and has roughly constant density, as implied for example by Eq. (6), then $\beta$ is overestimated by a factor of 2.7. This shows the importance of taking the full three-dimensional clump structure into account when analyzing the rotational properties of molecular cloud cores. Both prestellar cores (i.e. Jeans-unstable gas clumps) and protostellar cores in our model typically have $\beta<0.3$ with similar distributions. Thus, $\beta$ stays mainly in the interval $[0,0.3]$ and in this sense it remains approximately constant during the collapse.

It should be noted in passing that we also looked at the density structure of purely hydrodynamic turbulence, i.e. without self-gravity. If we again perform a clump-decomposition of the density structure and compute hypothetical $\beta$-values, we find $\beta \approx 2$. This is indicative of the high degree of vorticity inherent in all turbulent flows. However, it also suggests that dense molecular cloud cores are strongly influenced by self-gravity. The fact that all cores in the observational sample have $\beta<0.2$ implies that gravitational contraction is needed to achieve density contrasts high enough for sufficiently low $\beta$. This agrees with the picture of gravoturbulent fragmentation where molecular cloud structure as whole is dominated by supersonic turbulence but stars can only form in those regions where gravity overwhelms all other forms of support.

Comparing the two fits in Fig. 4 shows that our first set of assumptions is a better representation of the data. This is especially true during the early accretion phase where we have good statistics. It applies to different turbulent cloud environments as well. We conclude that - in a statistical sense - the angular momentum evolution of collapsing cloud cores can be approximately described as contraction of initially constant-density spheres undergoing rigid body rotation with constant angular velocity. This is consistent with the fact that cores from gravoturbulent fragmentation follow a Bonnor-Ebert-type radial density profile (Ballesteros-Paredes et al. 2003) and have roughly constant density in their innermost regions. It also supports the assumptions adopted by Goodman et al. (1993) and Burkert \& Bodenheimer (2000).

\subsection{Dependence of the specific angular momentum on the environment}

When we compare the results of our complete suite of numerical models (see Table 1) we find as a general trend that the average angular momentum falls with increasing Mach number. This is illustrated in Fig. 6a. However, this follows mainly 


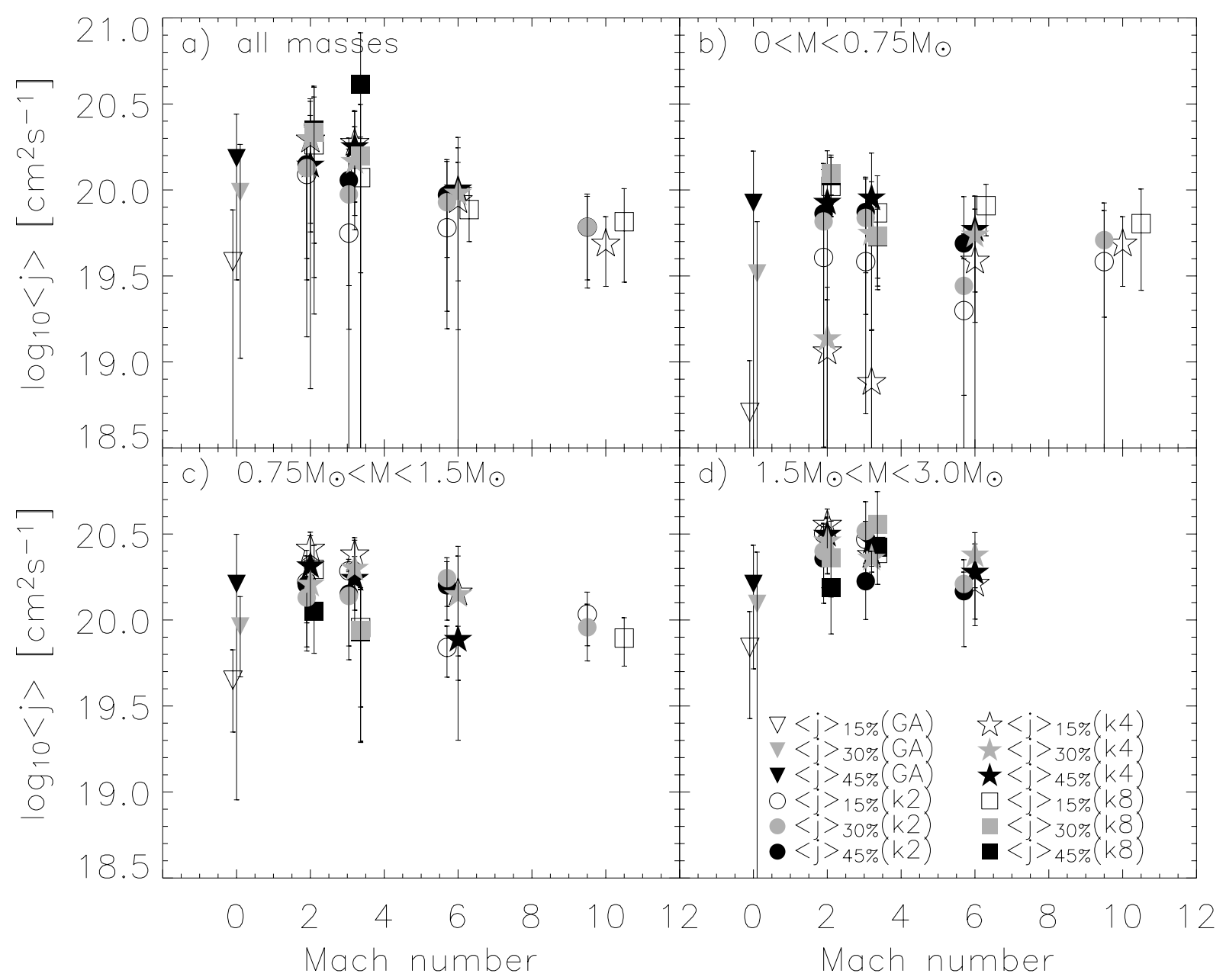

Fig. 6. Average specific angular momentum of protostellar objects in different turbulent environments as a function of the associated Mach number. Different shapes mark different driving scales $k$ (circle $-k=2.0$, star $-k=4.0$, square $-k=8.0$ ). GA stands for the Gaussian collapse without driving (arrow pointing downward). Different shades represent different stages of accretion (white - 15\% material accreted, gray - 30\% material accreted, black - 45\% material accreted). In a) all protostars (identified as "sink particle" in the simulations) were used in calculating the average, in b) through d) only objects in the denoted mass bins were considered. The error bars show the standard deviation of $\langle j\rangle$. For more clarity the symbols are distributed around the corresponding Mach number $\mathcal{M}$ with $\Delta \mathcal{M} / \mathcal{M}=5 \%$.

from the positive dependence of angular momentum on mass and from a correlation between average mass of the cloud cores and Mach number. As seen in Sect. 4.2 the specific angular momentum increases on average as the mass of the core rises. In environments with a low Mach number the mass growth of the cores is undisturbed over longer periods of time and so larger masses can accumulate. This can be inferred from Table 1, where we list both the number of cores and the accreted mass. For higher Mach numbers more cores form with on average less mass. Thus, the average angular momentum is expected to decrease with increasing Mach number (Fig. 6a).

To detect a direct dependence of the specific angular momentum on the Mach number we select cores that belong in a certain mass bin and average the specific angular momentum only over those cores. The results are shown in Figs. 6b-6d. We find that there is in general little spread of specific angular momentum for different scales and different times in accretion history independently of the Mach number. Low mass protostars (Fig. 6b) are an exception, in low Mach number environments they show especially low angular momentum in the early accretion phase. However, within the error bars we do not find a further dependence of $j$ on the Mach number.

Compared to the Gaussian collapse case, turbulent driving with small Mach numbers results in higher specific angular momenta. This is due to input of turbulent energy that can be converted into rotational energy if the turbulent velocities are not too high.

\section{Orientation of angular momentum vector}

We find in our simulations that neighboring protostellar cores have similarly oriented angular momenta. In Fig. 7 the correlation of specific angular momenta of different protostellar cores with respect to their orientations is shown as a function of distance. We calculate the scalar product $\boldsymbol{j} \cdot \boldsymbol{j}$ and average over cores with similar distances.

Figure $8 \mathrm{a}$ shows the spatial configuration in model M6k2 after $15 \%$ of the available material has been accreted onto the protostellar cores. These cores form in small aggregates with diameters below 0.07 pc. The corresponding Fig. 7a shows a 


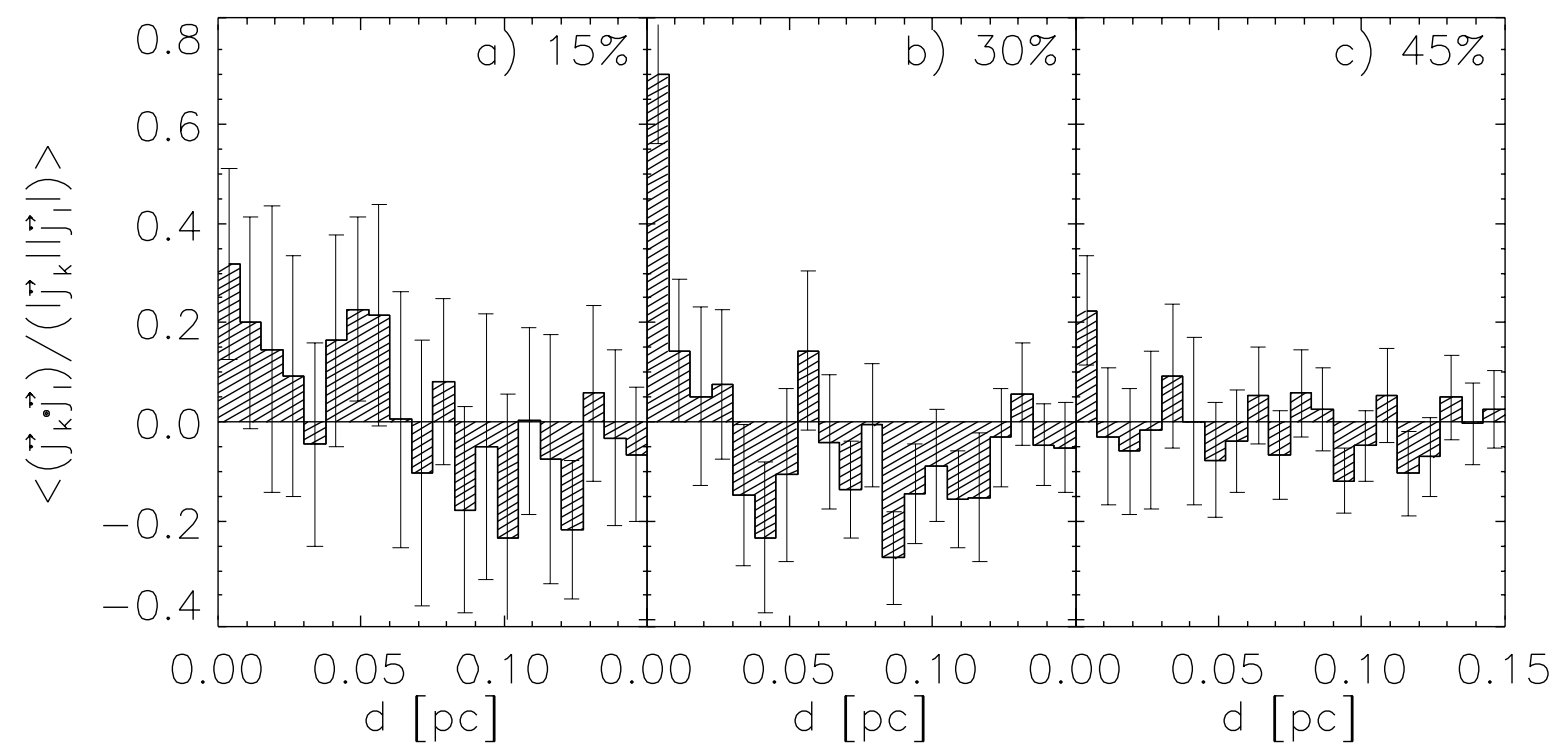

Fig. 7. The correlation of specific angular momenta of different protostellar cores in model M6k2 with respect to their orientation as a function of distance between the cores. As a measure for the correlation the scalar product of different cores was taken and averaged over cores that exhibit similar distances between each other. High positive values denote co-aligned and high negative values denote anti-aligned angular momenta. The three graphs a)-c) show three different times at which $15 \%, 30 \%$ and $45 \%$ of the available material was accreted. The error bars show the standard deviation of the averaged correlations.

spatial correlation of the specific angular momenta for small distances. The correlation length is approximately $0.05 \mathrm{pc}$. Thus, correlation length and cloud size are closely connected. This can be understood because within one molecular cloud clump neighboring cores accrete from the same reservoir of gas and consequently gain similar specific angular momentum. In the early phase of accretion we therefore expect disks and protostellar outflows of neighboring protostars to be closely aligned.

Indeed, several examples of parallel disks and outflows have been reported in low-mass, isolated Bok globules by Froebrich \& Scholz (2003), Kamazaki et al. (2003), Nisini et al. (2001), and Saito et al. (1995). Alternative explanations for the alignment of the symmetry axes of young stars include density gradients in the prestellar phase or the presence of strong magnetic fields. However, Duchêne \& Ménard (2003) found that the disks of T-Tauri stars driving jets or outflows are perpendicular to the magnetic field but disks of T-Tauri stars without jet are parallel to the field lines. This is very puzzling, showing the complexity of the situation that will naturally arise in strongly turbulent flows.

During subsequent accretion the correlation length decreases to values below $0.015 \mathrm{pc}$ (Fig. $7 \mathrm{~b}$ ). This means that only close systems remain correlated (see also Fig. 8b). This has three reasons. First, small $N$ systems of embedded cores are likely to dissolve quickly as close encounters lead to ejection (e.g., Reipurth \& Clarke 2001). Only close binaries are able to survive for a long time (e.g., Kroupa 1995a,b). The correlation length therefore decreases with time. Second, the same turbulent flow that generated a collapsing high-density clump in the first place may also disrupt it again before it is fully accreted. If the clump contains several protostars they will disperse, again decreasing the correlation. Third, the opposite may happen. Turbulence may bring in fresh gas. The protostars are then able to continue accretion, but the specific angular momentum of the new matter is likely to be quite different from the original material. As protostars accrete at different rates, we expect a spread in $\boldsymbol{j}$ to build up and the alignment will disappear. At later stages of the evolution, we expect that the correlation between the specific angular momenta of close protostellar objects has disappeared almost completely. This is evident in Fig. 7c. Furthermore, Fig. 8c demonstrates that most of the initial subclustering has disappeared by then.

\section{Summary and conclusions}

We studied the rotational properties and time evolution of the specific angular momentum of prestellar and protostellar cores formed from gravoturbulent fragmentation in numerical models of supersonically turbulent, self-gravitating molecular clouds. We considered rms Mach numbers ranging from 2 to 10 , and turbulence that is driven on small, intermediate, and large scales, as well as one model of collapse from Gaussian density fluctuations without any turbulence. Our sample thus covers a wide range of properties observed in Galactic starforming regions, however, our main focus lies in typical lowto intermediate-mass star-forming regions like $\rho$-Ophiuchi or Taurus.

With the appropriate physical scaling, we find the specific angular momentum $j$ of prestellar cores in our models, i.e. cloud cores as yet without central protostar, to be on average $\langle j\rangle=7 \times 10^{20} \mathrm{~cm}^{2} \mathrm{~s}^{-1}$. This agrees remarkably well with observations of cloud cores by Caselli et al. (2002) or Goodman et al. (1993). Some prestellar cores go into collapse to build up stars and stellar systems. The resulting protostellar objects have on average $\langle j\rangle=8 \times 10^{19} \mathrm{~cm}^{2} \mathrm{~s}^{-1}$. This is one order of 

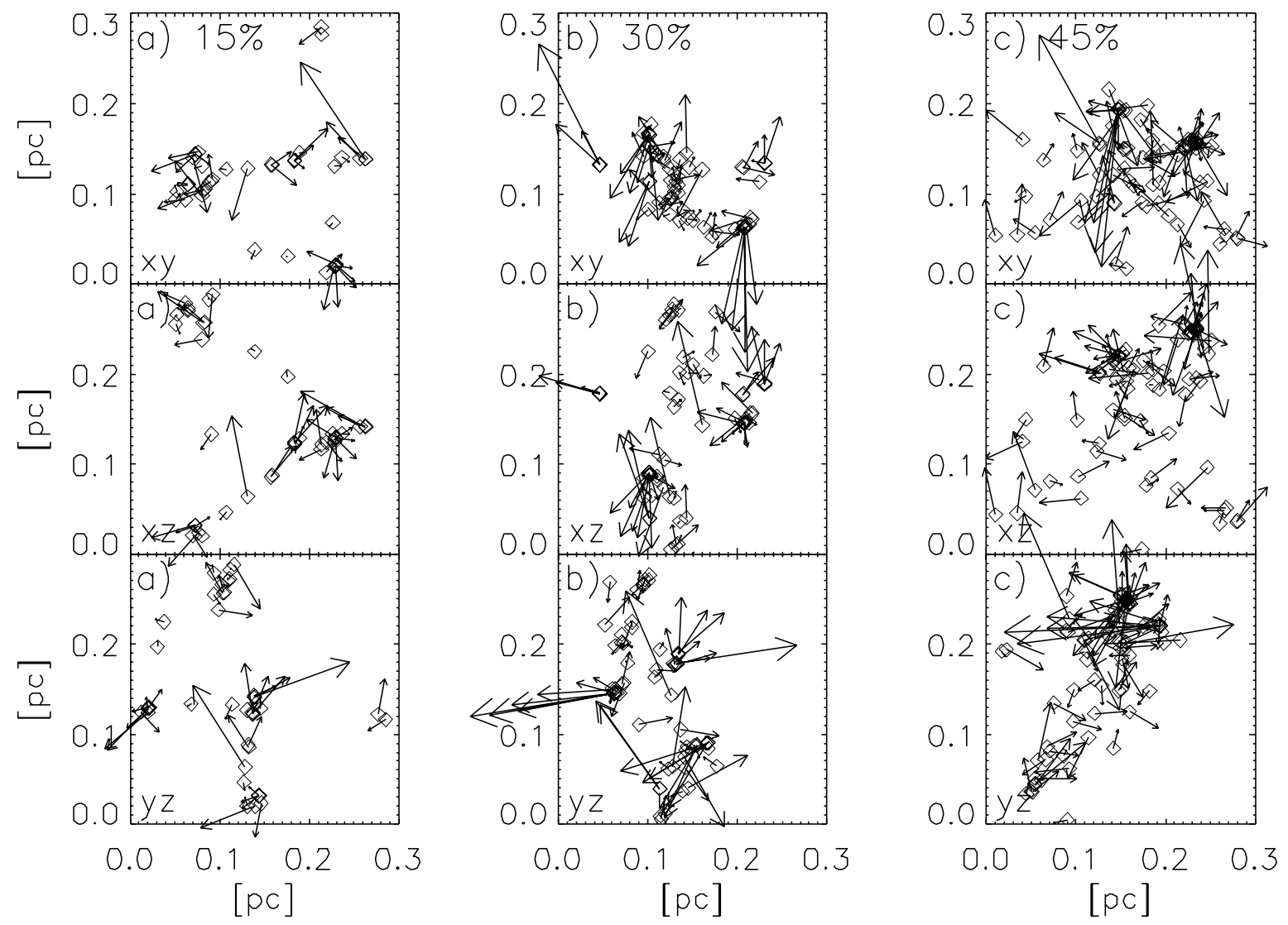

Fig. 8. Orientation of the angular momenta (arrows) and spatial distribution of the protostellar cores (diamonds) that formed in model M6k2. We present the projections on the $x y, x z$ and $y z$-plane. The spatial distributions are compared for three different times at which $15 \%$ a), $30 \%$ b) and $45 \%$ c) of the available material is accreted. The length of the arrows scales with the specific angular momentum of the protostellar core.

magnitude less, and falls into the range observed in G-dwarf binaries (Duquennoy \& Mayor 1991). Collapse induced by gravoturbulent fragmentation is accompanied by a substantial loss of specific angular momentum. This is mostly due to gravitational torque exerted by the ambient turbulent flow and to close encounters occurring when the protostars are embedded in dense clusters. This eases the "angular momentum problem" in star formation without invoking the presence of strong magnetic fields.

The time evolution of $j$ is intimately connected to the mass accretion history of a protostellar core. As interstellar turbulence and mutual interaction in dense clusters are highly stochastic processes, the mass growth of individual protostars is unpredictable and can be very complex. In addition, a collapsing cloud core can fragment further into a binary or higherorder multiple or evolve into a protostar with a stable accretion disk. It is the ratio of rotational to gravitational energy $\beta$ that determines which route the object will take. This is seen in the turbulent cloud cores studied here as well as in simulations of isolated cores where magnetic fields are important (e.g., Boss 1999). The $\beta$-distribution resulting from gravoturbulent cloud fragmentation reported here agrees well with $\beta$-values derived from observations (Goodman et al. 1993). The average value is $\beta \approx 0.05$. Note that we find that the distribution of $\beta$ stays essentially the same during collapse and accretion (see also Burkert \& Bodenheimer 2000; Goodman et al. 1993).
Although the accretion history and thus the evolution of the specific angular momentum of a single protostellar object is complex, we find a clear correlation between $j$ and mass $M$. This can be interpreted conveniently assuming collapse of an initially uniform density sphere in solid body rotation. Our models of gravoturbulent cloud fragmentation are best represented by the relation $j \propto M^{2 / 3}$.

When prestellar cores form by compression as part of supersonically turbulent flows and then go into collapse and possibly break apart into several fragments due to the continuing perturbation by their turbulent environment, we expect neighboring protostars to have similarly oriented angular momentum, at least during their early phases of accretion. Star clusters form hierarchically structured, with several young stellar objects being embedded in the same clump of molecular cloud material. These protostars accrete from one common reservoir of gas and consequently gain similar specific angular momentum. Their disks and protostellar outflows therefore will closely align. Indeed, there are several examples of parallel disks and outflows seen in low-mass, isolated Bok globules (Froebrich \& Scholz 2003; Kamazaki et al. 2003; Nisini et al. 2001; Saito et al. 1995). During later phases of cluster formation, the initial substructure becomes erased by dynamical effects and the correlation between the angular momenta of neighboring protostars vanishes. This is in agreement with our numerical calculations of gravoturbulent cloud fragmentation. They show small 
groups of close protostellar objects that have almost aligned specific angular momenta. As expected, the alignment occurs during the early phase of accretion as neighboring protostars accrete material from the same region with similar angular momentum. During the subsequent evolution the correlation length decreases. This is either because protostellar aggregates disperse, or because infalling new material with different angular momentum becomes distributed unevenly among the protostars.

Altogether, the process of gravoturbulent fragmentation, i.e. the interplay between supersonic turbulence and selfgravity of the interstellar gas, constitutes an attractive base for a unified theory of star formation that is able to explain and reproduce many of the observed features in Galactic star forming regions (Mac Low \& Klessen 2004). Our current study contributes with a detailed analysis of the angular momentum evolution during collapse.

Acknowledgements. We thank Peter Bodenheimer and Mordecai Mac Low for numerous stimulating discussions, and we thank our referee for insightful comments and suggestions. A.K.J. and R.S.K. acknowledge support by the Emmy Noether Program of the Deutsche Forschungsgemeinschaft (grant No. KL1358/1).

\section{References}

Ballesteros-Paredes, J., Hartmann, L., \& Vázquez-Semadeni, E. 1999a, ApJ, 527, 285

Ballesteros-Paredes, J., Vázquez-Semadeni, E., \& Scalo, J. 1999b, ApJ, 515, 286

Ballesteros-Paredes, J., Klessen, R. S., \& Vázquez-Semadeni, E. 2003, ApJ, 592, 188

Barranco, J. A., \& Goodman, A. A. 1998, ApJ, 504, 207

Bate, M. R., \& Burkert, A. 1997, MNRAS, 288, 1060

Bate, M. R., Bonnell, I. A., \& Price, N. M. 1995, MNRAS, 277, 362

Benz, W. 1990, in Numerical Modelling of Nonlinear Stellar Pulsations Problems and Prospects (Dordrecht: Kluwer), 269

Bodenheimer, P. 1995, ARA\&A, 33, 199

Bodenheimer, P., Burkert, A., Klein, R. I., \& Boss, A. P. 2000, Protostars and Planets IV, ed. V. Mannings, A. P. Boss, \& S. S. Russell (Tucson: Univ. of Arizona Press), 675

Boss, A. P. 1999, ApJ, 520, 744

Burkert, A., \& Bodenheimer, P. 2000, ApJ, 543, 822

Caselli, P., Benson, P. J., Myers, P. C., \& Tafalla, M. 2002, ApJ, 572, 238

Duchêne, G., \& Ménard, F. 2003 [arXiv: astro-ph/0306379]

Duquennoy, A., \& Mayor, M. 1991, A\&A, 248, 485

Ebert, R., von Hoerner, S., \& Temesváry, S. 1960, Die Entstehung von Sternen durch Kondensation diffuser Materie (Berlin: SpringerVerlag), 315

Elmegreen, B. G. 1993, ApJ, 419, L29

Fisher, R. T. 2004, ApJ, 600, 769

Froebrich, D., \& Scholz, A. 2003, A\&A, 407, 207

Gammie, C. F., Lin, Y., Stone, J. M., \& Ostriker, E. C. 2003, ApJ, 592, 203

Goodman, A. A., Benson, P. J., Fuller, G. A., \& Myers, P. C. 1993, ApJ, 406, 528

Halbwachs, J. L., Mayor, M., Udry, S., \& Arenou, F. 2003, A\&A, 397, 159
Heitsch, F., Mac Low, M.-M., \& Klessen, R. S. 2001, ApJ, 547, 280

Hunter, J. H., \& Fleck, R. C. 1982, ApJ, 256, 505

Jijina, J., Myers, P. C., \& Adams, F. C. 1999, ApJS, 125, 161

Kamazaki, T., Saito, M., Hirano, N., Umemoto, T., \& Kawabe, R. 2003, ApJ, 584, 357

Klessen, R. 1997, MNRAS, 292, 11

Klessen, R. S. 2001a, ApJ, 556, 837

Klessen, R. S. 2001b, ApJ, 550, L77

Klessen, R. S., \& Burkert, A. 2000, ApJS, 128, 287

Klessen, R. S., \& Burkert, A. 2001, ApJ, 549, 386

Klessen, R. S., Heitsch, F., \& Mac Low, M.-M. 2000, ApJ, 535, 887

Kroupa, P. 1995a, MNRAS, 277, 1491

Kroupa, P. 1995b, MNRAS, 277, 1507

Larson, R. B. 1995, MNRAS, 272, 213

Larson, R. B. 2003, Rep. Progress Phys., 66, 1651

Li, P. S., Norman, M. L., Mac Low, M.-M., \& Heitsch, F. 2004, ApJ, 605,800

Lin, D. N. C., \& Papaloizou, J. C. B. 1996, ARA\&A, 34, 703

Lombardi, J. C., Sills, A., Rasio, F. A., \& Shapiro, S. L. 1999, J. Comp. Phys., 152, 687

Mac Low, M.-M. 1999, ApJ, 524, 169

Mac Low, M.-M., \& Klessen, R. S. 2004, Rev. Mod. Phys., 76, 125

Mac Low, M.-M., Klessen, R. S., Burkert, A., \& Smith, M. D. 1998, Phys. Rev. Lett., 80, 2754

Mathieu, R. D., Ghez, A. M., Jensen, E. L. N., \& Simon, M. 2000, Protostars and Planets IV, ed. V. Mannings, A. P. Boss, \& S. S. Russell (Tucson: Univ. of Arizona Press), 703

Monaghan, J. J. 1992, ARA\&A, 30, 543

Motte, F., André, P., \& Neri, R. 1998, A\&A, 336, 150

Mouschovias, T. C., \& Paleologou, E. V. 1979, ApJ, 230, 204

Mouschovias, T. C., \& Paleologou, E. V. 1980, ApJ, 237, 877

Nakano, T. 1998, ApJ, 494, 587

Nisini, B., Massi, F., Vitali, F., et al. 2001, A\&A, 376, 553

Padoan, P. 1995, MNRAS, 277, 377

Padoan, P., \& Nordlund, Å. 1999, ApJ, 526, 279

Padoan, P., \& Nordlund, A.. 2002, ApJ, 576, 870

Papaloizou, J. C. B., \& Lin, D. N. C. 1995, ARA\&A, 33, 505

Pirogov, L., Zinchenko, I., Caselli, P., Johansson, L. E. B., \& Myers, P. C. $2003, A \& A, 405,639$

Reipurth, B., \& Clarke, C. 2001, AJ, 122, 432

Saito, M., Kawabe, R., Ishiguro, M., et al. 1995, ApJ, 453, 384

Shu, F. H. 1977, ApJ, 214, 488

Shu, F. H., Adams, F. C., \& Lizano, S. 1987, ARA\&A, 25, 23

Simon, M. 1992, in Complementary approaches to double and multiple star research, ASP Conf. Ser., 32, IAU Coll., 135, 41

Spitzer, L. 1968, Diffuse matter in space (New York: Interscience Publication)

Stone, J. M., Ostriker, E. C., \& Gammie, C. F. 1998, ApJ, 508, L99

Vázquez-Semadeni, E., Ballesteros-Paredes, J., \& Klessen, R. S. 2003, ApJ, 585, L131

Vázquez-Semadeni, E., Ostriker, E. C., Passot, T., Gammie, C. F., \& Stone, J. M. 2000, Protostars and Planets IV, ed. V. Mannings, A. P. Boss, \& S. S. Russell (Tucson: Univ. of Arizona Press), 3

Whitworth, A., \& Summers, D. 1985, MNRAS, 214, 1

Whitworth, A. P., Bhattal, A. S., Francis, N., \& Watkins, S. J. 1996, MNRAS, 283, 1061

Williams, J. P., Blitz, L., \& McKee, C. F. 2000, Protostars and Planets IV, ed. V. Mannings, A. P. Boss, \& S. S. Russell (Tucson: Univ. of Arizona Press), 97 